Case Report

\title{
Electroacupuncture for Decorticate Rigidity of the Upper Limbs in a Patient with Anoxic Brain Damage
}

\author{
WooSang Jung, ${ }^{1}$ SeungWon Kwon, ${ }^{1}$ SeongUk Park, ${ }^{2}$ SangKwan Moon, ${ }^{1}$ \\ JungMi Park, ${ }^{2}$ ChangNam Ko, ${ }^{2}$ and KiHo Cho ${ }^{1}$ \\ ${ }^{1}$ Department of Cardiovascular and Neurological Diseases, Kyung Hee University Oriental Medicine Hospital, \\ 1 Hoegi-dong, Dongdaemun-gu, Seoul 130-702, Republic of Korea \\ ${ }^{2}$ Department of Cardiovascular and Neurologic Diseases, Kyung Hee University Hospital at Gangdong, 149, \\ Sangil-dong, Seoul, Republic of Korea
}

Correspondence should be addressed to SeungWon Kwon; kkokkottung@hanmail.net

Received 12 May 2013; Accepted 31 July 2013

Academic Editor: Masahiro Kohzuki

Copyright (c) 2013 WooSang Jung et al. This is an open access article distributed under the Creative Commons Attribution License, which permits unrestricted use, distribution, and reproduction in any medium, provided the original work is properly cited.

\begin{abstract}
Decorticate rigidity is a type of abnormal posturing that can make it difficult to move a patient and to change the patient's position to prevent a decubitus ulcer. This condition poses a latent risk of bed sores. To prevent those complications, we used electroacupuncutre for decorticate rigidity of the upper limbs in a patient with anoxic brain damage. A 51-year-old man complained of rigidity of both of the upper and lower extremities due to anoxic brain damage. His rigidity was exhibited as flexed arms and extended legs, which are the typical positions in decorticate rigidity. Prior to electroacupuncture, his decorticate rigidity was treated with dantrolene sodium and baclofen. However, his rigidity had not improved. This patient received total 41 sessions of electroacupuncture. The patient's Modified Ashworth's Scale changed from 4 at baseline to 2 after the treatment, indicating an improvement in the rigidity of the upper limbs. Preston's Hypertonicity Scale and passive ROM of the elbow joints also improved. We report the observed effects of electroacupuncture for decorticate rigidity of the upper limbs in a patient with anoxic brain damage. Further controlled studies are needed to determine whether electroacupuncture is a useful alternative treatment for decorticate rigidity in patients with anoxic brain damage.
\end{abstract}

\section{Introduction}

We report a case of decorticated rigidity due to anoxic brain damage that was successfully treated with electro-acupuncture combined with conventional therapeutic options such as muscle relaxants and physical manual therapy.

\section{Case History}

2.1. Patient Characteristics and Diagnosis. A 51-year-old man complaining of rigidity of both of the upper and lower extremities due to anoxic brain damage was admitted to the Department of Cardiovascular and Neurological Diseases of Kyung Hee University Oriental Medicine Hospital. This patient had collapsed during a marathon and had received cardiopulmonary resuscitation (CPR). The doctors recognized acute myocardial infarction (MI) and provided conventional therapies for this condition. However, anoxic brain damage was caused by insufficient blood supply to the brain. He manifested rigidity, dyspnea, and dysphagia. His rigidity was exhibited as flexed arms and extended legs, which are the typical positions in decorticate rigidity. Although this patient had underlying hypertension and diabetes mellitus, he and his family had not recognized these conditions until onset of MI. At admission, he revealed GCS score 7 (E4 V0 M3) and used tracheostomy tube, nasogastric tube. The brain computed tomography (CT) image taken at admission (Figure 1) revealed severe cerebral cortical atrophy. Laboratory data did not reveal abnormal values for all items. He was admitted to Kyung Hee University Oriental Medicine Hospital 3 months after onset. Prior to his admission, his decorticate rigidity was 
TABLE 1: The Modified Ashworth's Scale for grading spasticity [1].

\begin{tabular}{ll}
\hline Grade & Definition \\
\hline G0 & $\begin{array}{l}\text { No increase in muscle tone. } \\
\text { Slight increase in muscle tone, manifested by a catch } \\
\text { and release or by minimal resistance at the end of the } \\
\text { range of motion when the affected part(s) is moved in } \\
\text { flexion or extension. }\end{array}$ \\
& $\begin{array}{l}\text { Slight increase in muscle tone, manifested by a catch, } \\
\text { followed by minimal resistance throughout the } \\
\text { reminder (less than half) of the ROM. }\end{array}$ \\
G1+ & $\begin{array}{l}\text { More marked increase in muscle tone through most of } \\
\text { the ROM, but affected part(s) easily moved. }\end{array}$ \\
G2 & $\begin{array}{l}\text { Considerable increase in muscle tone passive } \\
\text { movement difficult. }\end{array}$ \\
Affected part(s) rigid in flexion or extension.
\end{tabular}

treated with dantrolene sodium (25 mg) and baclofen ( $5 \mathrm{mg}$ ). However, his rigidity had not improved, and his condition had remained the same since onset.

2.2. Treatment. Electro-acupuncture was performed as a therapy for symptoms of decorticated rigidity of the upper limbs. Daily electro-acupuncture treatment was performed between 14:00 and 15:00 for 41 consecutive days (PG-306, Japan). The acupoints used in this case were LI11, LI10, TE5, and LI4, bilaterally. The following conditions were used: stimulation wave, biphasic $(60 \mathrm{~Hz})$; pulse duration, $0.4 \mathrm{~ms}$; and stimulation strength, low. The electrical stimulation power was the same for each treatment (Pulse Generator PG-306, Suzuki Inc., Japan). The patient's elbow joints and wrists showed mild movement during electro-acupuncture therapy. Each treatment procedure lasted $20 \mathrm{~min}$ and was performed by skilled specialists (Korean Medicine Doctor (KMD)) by using stainless steel acupuncture needles (Dongbang Acupuncture Inc., Korea; $40 \mathrm{~mm}$ (length) $\times 0.25 \mathrm{~mm}$ (diameter)). Furthermore, Bojungikgitang, a Korean herbal medicine decoction, is administered to control general condition of this patient. There was no change in total daily dose of dantrolene sodium and baclofen before and after electroacupuncture treatment.

2.3. Evaluation Methods. We used 3 methods to evaluate the effect of electro-acupuncture on the patient's rigidity: the Modified Ashworth's Scale (MAS) (Table 1) [1], Preston's Hypertonicity Scale (Table 2) [2], and the measurement of the passive range of motion (ROM) of the elbow joints.

2.4. Course of Symptoms. The patient was treated for 41 days, and the results are presented graphically in Figures 1, 2, and 3. The initial MAS score of 4 improved slightly during the procedure: the first improvement in this score ( 3 points) was observed after the 6th treatment, and a further improvement to 2 points was noted by the time of the 24 th treatment. The MAS score remained at 2 points at the last session (Figure 2). The initial Preston's Hypertonicity Scale score of 3 fell to 2 points after the 6th treatment. Preston's Hypertonicity Scale
TAble 2: Preston's Hypertonicity Scale [2].

\begin{tabular}{ll}
\hline Grade & Definition \\
G0 & $\begin{array}{l}\text { No abnormal tone detected during slow, passive } \\
\text { movement. } \\
\text { Mild: first tone or resistance is felt when the muscle is } \\
\text { in a lengthened position during slow passive } \\
\text { movement. }\end{array}$ \\
G1 & $\begin{array}{l}\text { Moderate: first tone or resistance is felt in the } \\
\text { midrange of the muscle during slow passive } \\
\text { movement. } \\
\text { G2 }\end{array}$ \\
Gevere: first tone or resistance is felt when the muscle \\
is in a shortened range during slow passive movement.
\end{tabular}

score of 1 was noted at the time of the 24 th treatment, and this value remained unchanged until the last treatment (Figure 3).

Before treatment, the passive ROM of the left elbow joint was $10^{\circ}$, and that of right elbow joint was $0^{\circ}$. With continuous electro-acupuncture therapy, the passive ROM of both elbow joints improved. Approximately 24 days after initiating treatment, the passive ROM of left elbow joint increased to $180^{\circ}$ and the passive ROM of the right elbow joint increased to $90^{\circ}$. The passive ROMs of the left elbow joint and the right elbow joint remained constant at $180^{\circ}$ and $90^{\circ}$, respectively, until the last treatment (Figure 4).

\section{Discussion}

Decorticate rigidity is a type of abnormal posturing. Abnormal posturing is an involuntary flexion or extension of the arms and legs, indicating severe brain injury. Decorticate rigidity exhibited as flexion of the upper limbs and extension of the lower limbs indicates damage in the cerebral hemispheres, the internal capsule, and the thalamus. Our patient's rigidity was a result of anoxic brain damage due to myocardial infarction.

This patient exhibited flexed arms and extended legs, which are the typical positions in decorticated rigidity. This patient was diagnosed with anoxic brain damage on the basis of his medical history, symptoms, and brain imaging. Although he had been treated with conventional therapies for rigidity (muscle relaxants and physical therapy) after diagnosis, his symptoms did not improve before he was admitted to KyungHee University Oriental Medicine Hospital. The patient's family and caregiver had trouble in moving the patient owing to his severe rigidity. Furthermore, his rigidity made it difficult to change his position for preventing the formation of decubitus ulcers, and this patient was at latent risk of bedsores.

Several studies have shown that electro-acupuncture can treat rigidity [3, 4]. One of these studies [4] has suggested that high-frequency and low-strength electro-acupuncture on LI11, LI10, TE5, and LI4 can reduce rigidity of the upper limbs in stroke patients. Because the rigidity of the upper limbs of stroke patients exhibits a flexion, the authors of this study used acupoints LI11, LI10, TE5, and LI4 to stimulate the arm extensor muscle group, which is an antagonist of the arm flexor muscle group. Although the diagnosis of 


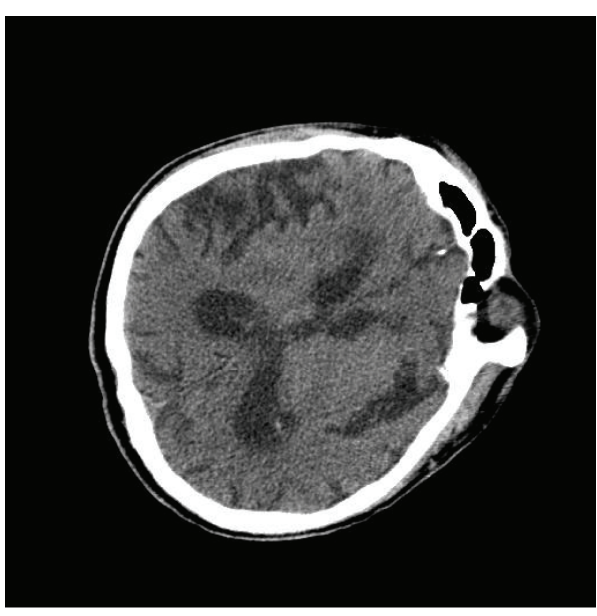

(a)

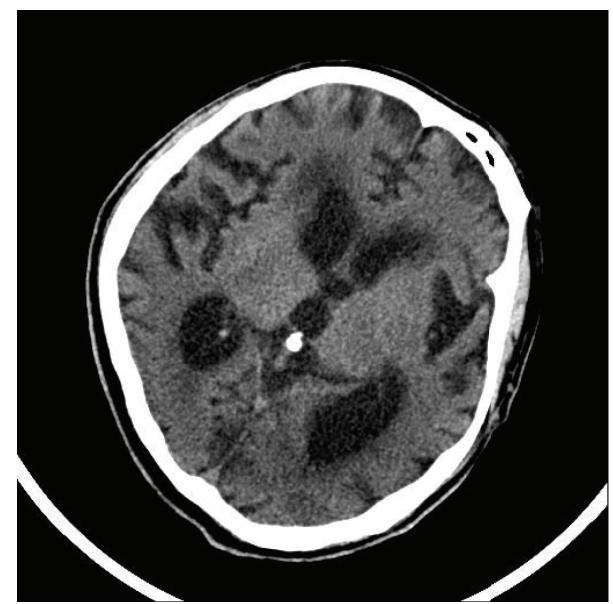

(b)

FIGURE 1: The brain computed tomography (CT) image at onset and admission time. (a) Brain CT image at onset and (b) brain CT image at admission time (3 months after onset).

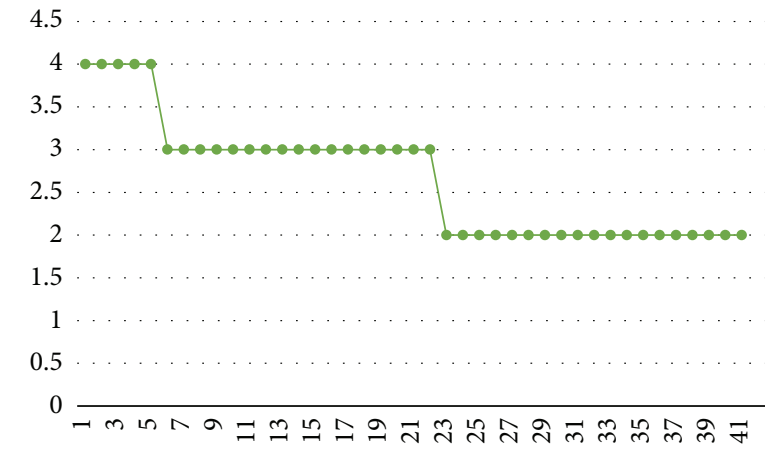

Figure 2: MAS score changes. MAS: Modified Ashworth's Scale; $X$ : numbers of sessions; $Y$ : MAS score.

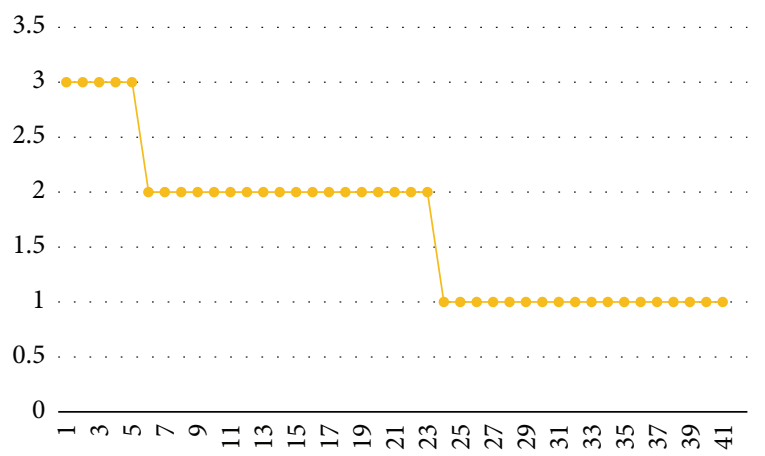

FIGURE 3: Preston's Hypertonicity Scale changes. $X$ : numbers of sessions; $Y$ : Preston's Hypertonicity Scale score.

the present case was anoxic brain damage, we observed a form of rigidity in this patient that was similar to that in stroke patients, namely, flexion of the upper limbs. As mentioned above, conventional therapy did not improve his rigidity. Thus, we used electro-acupuncture therapy based on the previous study in stroke patients (acupoints LI11, LI10,

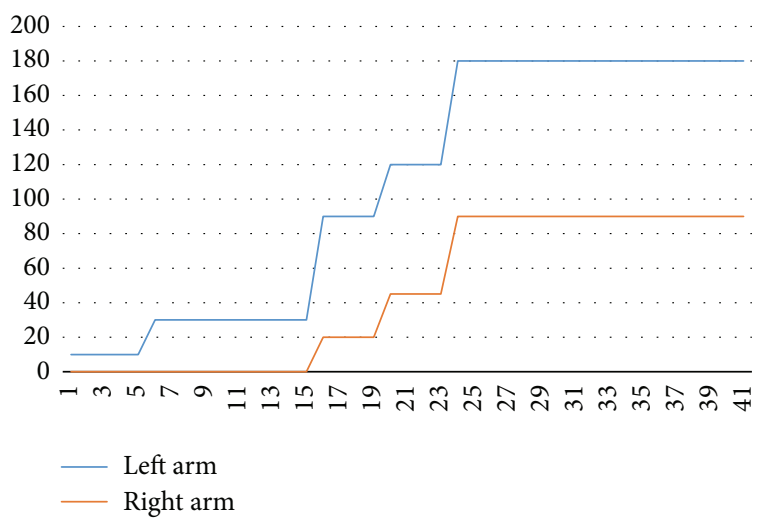

FIgURE 4: Passive ROM changes of both elbow joints. ROM: range of motion; $X$ : numbers of sessions; $Y$ : passive ROM of both elbows.

TE5, and LI4 and a high-frequency/low strength stimulation method $(60 \mathrm{~Hz}, 0.4 \mathrm{~ms}$ pulse duration, and low strength)) to relieve our patient's arm rigidity symptoms.

In the present study, the patient's upper limb rigidity gradually improved during the follow-up period. The MAS score and Preston's Hypertonicity Scale improved at both the 6 th and 24th sessions. The passive ROM of both elbow joints also improved during the 41 sessions, and notably, the passive ROM of the left elbow joint showed a normal value at the endpoint. Therefore, we believe that electro-acupuncture was effective for treating this patient's upper limb rigidity.

In general, anoxic brain damage which results in coma causes a vegetative state, and these patients will remain in a vegetative state until death. Therefore, prognosis of anoxic brain damage is very poor. And patients who reveal decorticate rigidity are in a coma or vegetative state and have poor prognoses, with risks for cardiac or respiratory arrest [5]. However, in this case, there were improvements in upper limbs rigidity due to anoxic brain damage. Thus, we could make it easy to move a patient and to change the patient's 
position to prevent a decubitus ulcer and related complications such as pneumonia. We think that the use of electroacupuncture for decorticate rigidity not only decreased tone of muscle of upper limbs but also prevented complications.

Baclofen is used to treat rigidity in conventional drug treatments $[6,7]$. However, patients who have nephropathy cannot use baclofen owing to its toxicity; moreover, a previous study [8] has suggested that patients on dialysis are exposed to nephrotoxicity during baclofen intake. Therefore, baclofen cannot be used to treat rigidity in patients who have nephropathy. We believe that electro-acupuncture may be an alternative for treating decorticate rigidity in elderly people undergoing dialysis. In our case, treatment with conventional therapy (drugs and physical therapy) for decorticate rigidity did not result in improvement, but improvement was noted in the rigidity of the upper limbs after treatment with electroacupuncture. Therefore, we propose that electro-acupuncture is a therapeutic option in patients who show no improvement despite therapy.

A limitation of the present case study is that we could not treat the rigidity of the lower limbs. The patient's lower limbs exhibited extension, but we could not find any studies in the literature that provided therapeutic methods for lowerlimb rigidity. In addition, because the symptoms of the lower limbs were less severe than those of the upper limbs at the time of admission, we concentrated our efforts on treating the rigidity of the upper limbs. Studies that provide data about treating lower-limb rigidity are required in the future. Furthermore, additional research is needed to determine the efficacy of electro-acupuncture on decorticate rigidity.

In summary, 41 days of electro-acupuncture treatment of LI11, LI10, TE5, and LI4 improved the rigidity of the upper limbs in patients with anoxic brain damage. Although it is difficult to draw conclusions based on a case study of only 1 patient, we propose that further studies would help determine whether electro-acupuncture is a plausible alternative in the treatment of decorticate rigidity of the upper limbs in patients with anoxic brain damage.

\section{Conflict of Interests}

The authors declare that they have no conflict of interests.

\section{References}

[1] R. W. Bohannon and M. B. Smith, "Interrater reliability of a modified Ashworth scale of muscle spasticity," Physical Therapy, vol. 67 , no. 2, pp. 206-207, 1987.

[2] H. Pendleton and W. Schultz-Krohn, Occupational Therapy, vol. 413, Mosby, 2006.

[3] S. K. Kang and Y. S. Kim, "Effectiveness of electro-acupuncture on spasticity in stroke patients," Orient Journal of Medicine, vol. 2, pp. 25-32, 1997.

[4] Y. S. Kim, "Antispastic effects of electro-acupuncture, TENS and NMES in stroke patient," Journal of Korean Acu-Puncture \& Moxibustion Society, vol. 17, pp. 209-220, 2000.

[5] M. Silverberg, M. Greenberg, and R. Hendrickson, Greenberg's Text-Atlas of Emergency Medicine, vol. 53, Lippincott Williams \& Wilkins, Philadelphia, Pa, USA, 2005.
[6] I. Richard and P. Menei, "Intrathecal baclofen in the treatment of spasticity, dystonia and vegetative disorders," Acta Neurochirurgica, Supplementum, no. 97, pp. s213-s218, 2007.

[7] C. R. Hansen, J. L. Gooch, and T. Such-Neibar, "Prolonged, severe intrathecal baclofen withdrawal syndrome: a case report," Archives of Physical Medicine and Rehabilitation, vol. 88, no. 11, pp. 1468-1471, 2007.

[8] A. El-Husseini, A. Sabucedo, J. Lamarche, C. Courville, and A. Peguero, "Baclofen toxicity in patients with advanced nephropathy: proposal for new labeling," American Journal of Nephrology, vol. 34, no. 6, pp. 491-495, 2011. 


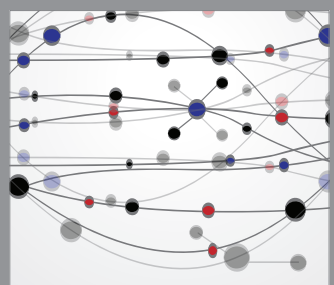

The Scientific World Journal
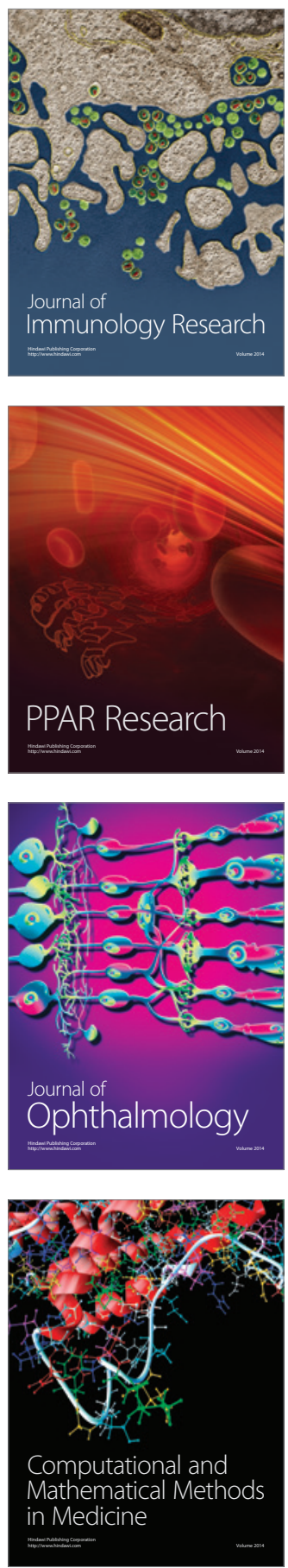

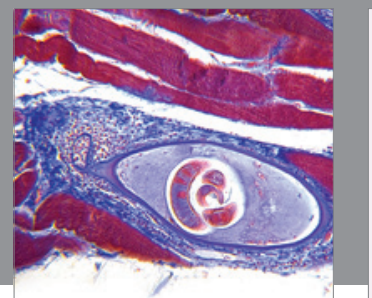

Gastroenterology

Research and Practice
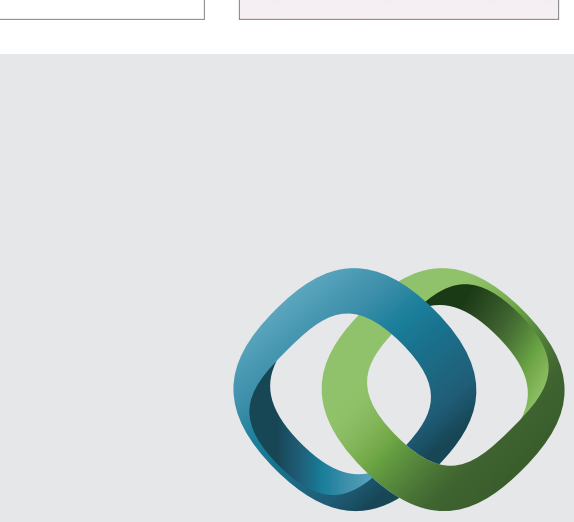

\section{Hindawi}

Submit your manuscripts at

http://www.hindawi.com
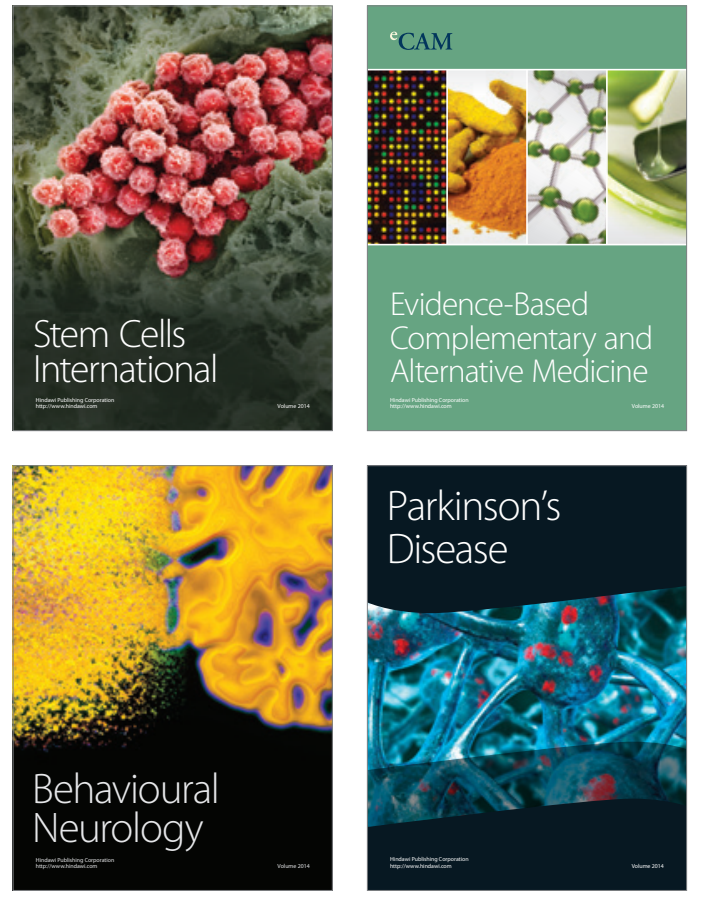
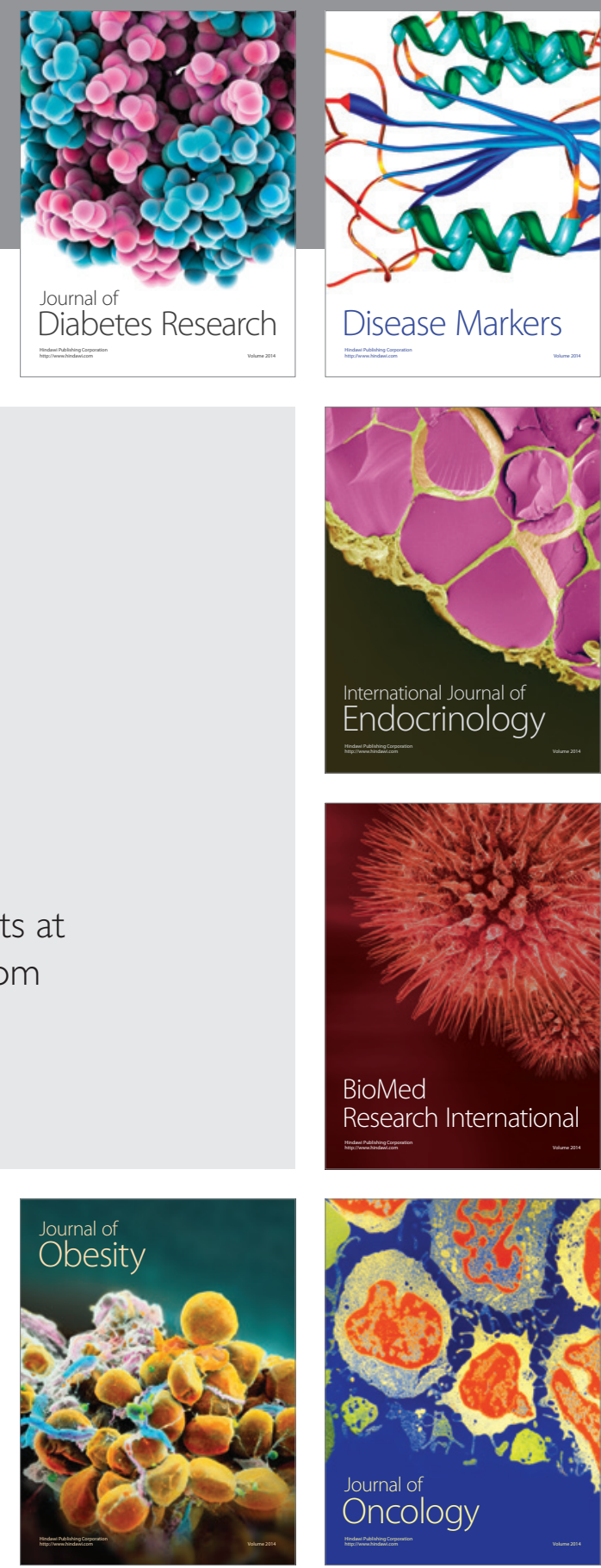

Disease Markers
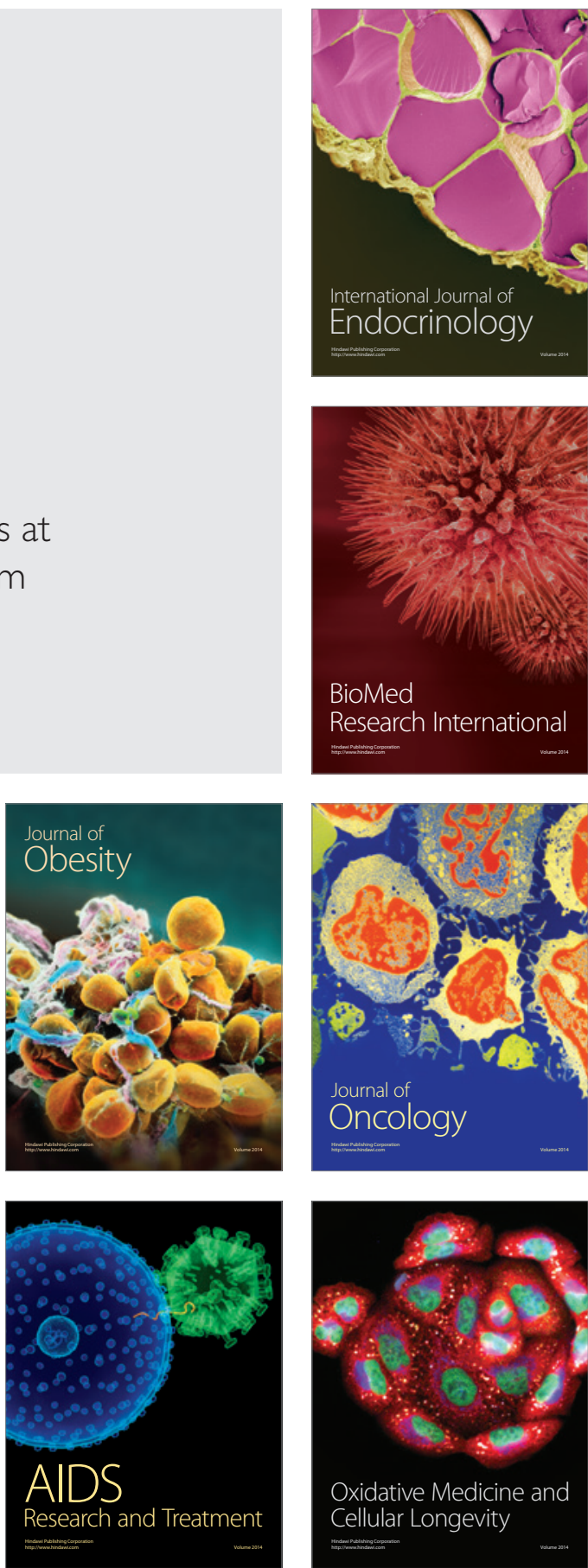